\title{
The Multiple Contexts of Brain Scaling: Phenotypic Integration in Brain and Behavioral Evolution
}

\author{
Barbara L. Finlay \\ Department of Psychology, Behavioral and Evolutionary Neuroscience Group, Cornell University, Ithaca, NY, USA
}

\section{Keywords}

Allometry of brain development · Developmental duration . Longevity

\begin{abstract}
Understanding the adaptive functions of increasing brain size have occupied scientists for decades. Here, taking the general perspective of the Extended Evolutionary Synthesis, the question of how brains change in size will be considered in two developmental frameworks. The first framework will consider the particular developmental mechanisms that control and generate brain mass, concentrating on neurogenesis in a comparative vertebrate context. The consequences of limited adult neurogenesis in mammals, and the dominating role of duration of neurogenesis for mammalian evolution will be discussed for the particular case of the teleost versus mammalian retina, and for paths of brain evolution more generally. The second framework examines brain mass in terms of life history, particularly the features of life history that correlate highly, if imperfectly, with brain mass, including duration of development to adolescence, duration of parental care, body and range size, and longevity. This covariation will be examined in light of current work on genetic causes and consequences of covariation in craniofacial bone groupings. The eventual development of a multivari-
\end{abstract}

ate structure for understanding brain evolution which specifically integrates formerly separate layers of analysis is the ultimate goal.

(c) 2022 S. Karger AG, Basel

\section{Introduction}

The framework of adaptation is central to understanding brain evolution, at every level. An adaptation in brain structure may allow an animal to compete better for resources in an existing niche, or create a new niche altogether. Establishing the mechanisms of basic features like motor agility or sensory discrimination makes sense as such features must underlie the general capacities of exploration, exploitation, and prediction essential for survival and reproduction. The brain itself is energetically expensive, and optimal configurations of circuits and networks might directly impact an animal's viability. Most work on such aspects of comparative brain structure and function as published in journals like Brain, Behavior and Evolution rarely tests the adaptive advantage of a change in a brain part explicitly. Examining the variations of structure and function of brains in a phylogenetic array, the significance of adaptation is usually implicit.

Correspondence to:

Barbara L. Finlay, blf2@ cornell.edu

Karger@karger.com www.karger.com/bbe

(C) 2022 S. Karger AG, Basel

Karger" 
When adaptation is considered explicitly, however, describing each brain change as an independent adaptation of a particular animal to an immediate environment quickly produces explanatory shortfalls. A daunting list of further considerations have been put forward. What follows here is an eclectic set of citations illustrating four such considerations: (1) phylogenetic history [Isler and Van Schaik, 2014], (2) comparisons of structure afforded by different environments [Olshausen and Field, 1996; de Visser and Krug, 2014; Lewicki et al., 2014; MacIver et al., 2017; Vermeij, 2017; MacIver and Finlay, 2021], (3) available developmental mechanisms, ranging from genomic construction to environmental and cultural instruction [Wilson and Sober, 1994; West-Eberhard, 2003; Wilson et al., 2008; Lai et al., 2019; Frankenhuis and Walasek, 2020; Hu et al., 2020], and finally, (4) the rules, heuristics, and variational structure embedded in genomic, physiological and computational mechanisms [Jablonka and Lamb, 2005; Newman, 2014; Noble et al., 2014; Holland et al., 2017; Darbellay et al., 2019; Slodkowicz and Goldman, 2020]. The contribution any feature makes to survival and reproduction (its adaptive value) is not displaced by these considerations, but contextualized. In the Extended Evolutionary Synthesis, Laland et al. [2015] argue that in order to have a full understanding of why any genotype persists or a phenotype presents as it does, these kinds of phylogenetic, environmental, developmental, and mechanistic considerations must also take their places.

Such sweeping injunctions must show they have interesting or useful consequences for evolutionary explanations. In this paper, we will consider two linked examples of how multiple levels of analysis interact to limit or promote particular mechanisms or phenotypes from the perspective of "evo-devo," the study of the two-way interaction of development with evolution. We will discuss first the suite of developmental mechanisms that potentially control the number of neurons in the brain, and how that suite differs in major vertebrate taxonomic groups. Comparing bony fish, with lifetime neurogenesis, to mammals whose neurogenesis is restricted primarily to early development, the spatial positioning and densities of receptor classes like rods and cones though quite similar in final arrangement, is produced by very different developmental mechanisms. In the second example, mammalian dependence on duration of early neurogenesis for control of neuron number, proves to have unexpected consequences for features of life history, considering early learning and parental care, and longevity. Before launching these specific examples, it is useful to stand back and consider how the nature of explanation in several fields of biology, and in cognitive neuroscience particularly, has dramatically changed in the last century to understand how the Extended Evolutionary Synthesis fits a more general pattern.

Over the last 50 to 100 years, many of the subdisciplines relevant to brain evolution have changed in similar ways. Whether it be population genomics [Watanabe et al., 2019], control of gene expression in stem cells [Furusawa and Kaneko, 2012], resolution of phylogenies [Song et al., 2012], information coding in the brain and brain plasticity [Anderson, 2014], or population biology [Ramírez and Marshall, 2017], each field has moved independently in its understanding of how its domain "works," but on functionally similar paths. Collectively, they have moved past positing explicitly stated mechanisms containing identified elements combined linearly (to be described more explicitly below) toward the clearer, but less absolute probabilistic, multifactorial, networked mechanisms we can now describe. In this historical context, the Extended Evolutionary Synthesis as applied to brain evolution is an analogous operation over multiple, historically separate research fields. That is, brain evolution can employ the same multifactorial strategies applied to single levels of analysis to guide integration over multiple, historically defined separate disciplines [Parsons and Albertson, 2013; Laughlin and Messier, 2015; Ramaekers et al., 2019]. Most of these fields have developed virtually de novo over the past several decades, confronting gigantic assemblages of phenomena, inventing new descriptive languages, beginning from absent or inappropriate mathematics, and inventing and interpreting research tools on the fly.

For example, at the first description of the double helix, the first candidate for essential Darwinian variation for selection was thought to be random mutation and variation at the level of the single base pair (reviewed in Kirschner and Gerhart [2005]). In the first guesses of the relationship of genotype to phenotype, direct identity of elements was proposed across levels: "the gene" for eye color or for temperament, or in pathology, "the gene" for schizophrenia. Supraordinate structure was very soon discovered in genome variation, including duplications and repetitions of gene sequences from single elements to entire genomes; identification and reassignment of DNA segments from coding elements to regulatory elements in what was originally called "junk DNA"; description of genetic drift, gene drives; jumps and rearrangements; the role of the epigenome, and so on [Holland et al., 2017; Ramaekers et al., 2019; Watanabe et al., 2019].
Finlay 
In early neuroscience theories, a similar linear combinatorial logic was the first to be expressed. Single neurons were first taken as explicit units of meaning, representing particular entities or features in the world, like flies or edge detectors [Hubel and Wiesel, 1968]. Local cortical areas or other brain parts were identified as semi-encapsulated modules dedicated to explicit computations, such as syntactic language parsing or face identification [Damasio and Geschwind, 1984; Kanwisher et al., 1997]. Later, the distribution of a specified representation over multiple neurons began to be demonstrated, and imaging showed how any single cortical area might contribute to any number of sensory, motor, and cognitive functions [Anderson, 2014]. Most currently, network analyses of brain function, and coordination by oscillatory processes across regions were superimposed on and often entirely subsumed cortical areas as their relational "nodes" and "edges" [Sporns, 2011; Cohen and d'Esposito, 2016].

Thinking of these conceptually similar initial characterizations as "tactical" rather than describing them with pejoratives like "confused" or "oversimplified" allows us to see how we attempt to simplify complex domains, and more easily recognize them when the next difficult domain comes up. The progression of these ideas are tactical moves in making the intractable tractable. Any implication that we have now arrived, in any of these fields, at the "correct" understanding should be avoided, but better still, it would be best to have, if possible, consciousness of the tactical frameworks we employ, what they address and their limitations. The two following examples are both cases of intersections of explanatory domains of the causes and consequences of changing neuron number and brain mass, where, when relevant, for example, developmental duration may have consequences for multiple aspects of adult phenotypes.

\section{Intersections of Explanation Concerning Brain Size in Brain Evolution}

\section{Why Changing Developmental Duration Is the}

Principal Way of Changing Brain Mass in Mammals

In this case, change in mammalian brain mass is the outcome, and changed developmental duration is its reasonably well-supported mechanistic cause. The mechanistic contexts and constraints on brain mass and developmental duration all fall within the general domain of neuroembryological phenomenon:

a. Diffusing molecules are central to establishing embryonic axial polarity and regionalization. b. Late neurogenesis in mammals is rare, and of small magnitude.

c. Other candidate mechanisms than duration of neurogenesis to increase neuron number can be ruled out for multiple reasons.

Establishment of Axial Polarity and Regionalization with Diffusing Molecules

The first step of early specification and regionalization of the vertebrate embryo is accomplished by Hox genes specifying both its rostro-caudal polarity, and its ordered segmentation. Determination of axial positions is the first step of differentiation in the embryo, concurrent with segmentation, and antedates the specification of adult cell types: that is, the axes of the map are specified before the map contents. The relative levels of expression (not on/ off) of a complement of the eleven Hox genes in a progression from rostral to caudal fixes these features [Burke et al., 1995].

Establishment of the polarity of the neocortex is embedded in the establishment of the polarity of the whole embryo. First, the rostro-caudal polarity of the brain, and its segmentation are fixed [Puelles et al., 2013; Parker and Krumlauf, 2017]. In the forebrain, the pallial surface of the lateral ventricles gives rise to the cortex, and many non-cortical structures. Interestingly for this argument to be made about the importance of diffusible molecules in constraining the size of embryonic anlage, the dorsal pallium, the structure giving rise to the neocortex in mammals, is poorly defined in fish. Its ambiguous definition has been conjectured to arise from the failure of diffusible molecules released from the pallial margin to reach the centrally located dorsal pallium in the enlarging forebrain [Puelles, 2001, 2011; Striedter and Northcutt, 2020].

With the location of the cortical plate fixed, its polarity is established next. The cortical plate is embedded in multiple signaling pathways that progressively restrict or direct the competence of cells positioned along its major axes to respond to local or general signals [Rubenstein et al., 1998; Molnár et al., 2014; Ypsilanti and Rubenstein, 2016; Cadwell et al., 2019]. Signaling pathways have multiple media for communication, through direct cell-tocell coupling to diffusion through extracellular space. The rostrocaudal polarity of the cortical plate is established by diffusion of a morphogen, one of the fibroblast growth factors, expressed at the rostral part of the anterior neural ridge and diffusing from there. The level of the morphogen molecule, in its relevant context of other signaling gradients in the cortical plate [Balasubramanian and Zhang, 2016], causes a suite of transcription factors to be 
expressed in rostral-to-caudal gradients across the cortical plate. These in turn specify the rostrocaudal order of the somatomotor, auditory, and caudal visual primary cortices. This order defines or is the first index of cortical polarity [Assimacopoulos et al., 2012; Cadwell et al., 2019]. These cortical areas shortly generate their half of specific recognition molecules and receptors between each area and the reciprocals of the thalamic nucleus specific to each one [Anton-Bolanos et al., 2018]. Next, a rostral-to-caudal sweep in gene expression and in cortical maturation begins, an organizing program for the hierarchical organization of many features of the adult cortex [Bernard et al., 2012; Lein et al., 2015].

The FGF morphogen has its action as a diffusible molecule released from a rostral neural ridge location, initiated at the earliest possible time of cortical plate formation, approximately the time of the final division and differentiation of the first cortical neuroblast. At this point, the cortical plate can be identified, but is at its minimum size. The spatial range of such diffusible agents is severely limited, the more so in that it must establish a "readable" gradient amongst other spatially fixed or diffusible gradients, not an on/off signal to simply be detected [Balasubramanian and Zhang, 2016]. The most basic evidence in support of a size-limiting role of diffusible gradients in early embryogenesis is the equivalent size of embryos at equivalent maturational states, independent of their adult size - for example, at the developmental point in the rat and human brains when the lateral ventricles of the forebrain are conspicuous, and layer 6 of the cortex is in process of generation (14 and 50 days post-conception, respectively), both measure about $2 \mathrm{~mm}$ from the midline to the outer bound of the lateral ventricle, though the human brain will be approximately 650 times larger than the rat's at maturity [Workman et al., 2013]. We will cover a bit more detail on "size" in a later section.

\section{The Relative Absence of Late Neurogenesis in}

Mammals

Among vertebrates, only mammals restrict neurogenesis almost entirely to early development [Kaslin et al., 2008]. Sharks, rays, bony fish, and amphibia may generate neurons throughout their lifetimes, variously related to normal growth, environmental changes, sex or social status changes, and metamorphosis. In lizards and birds, neurogenesis is plentiful throughout most of the forebrain and most of the lifespan, though restricted otherwise, and is often seasonal. In birds, late neurogenesis has been highlighted for its role in the learning and performance of bird song [Alvarez-Buylla, 1992], and (argu- ably) its role in seasonal foraging [Smulders et al., 2010]. In mammals, by contrast, adult neurogenesis has only been verified for a small fraction of cells in the forebrain, one cohort migrating to replace particular neuron classes in the olfactory bulb, and a separate cohort contributing to neuron turnover in the hippocampus [Ming and Song, 2005]. Note that by "late neurogenesis" we do not mean any neurogenesis that occurs after birth, but neurogenesis occurring when the species in question is post-adolescent. In the developmentally altricial mouse and rat, immediately postnatal neurogenesis is fully continuous with neurogenesis that begins prenatally and concludes after birth, principally in the neocortex and cerebellum [Altman and Das, 1966].

Narrowing Candidate Mechanisms for Evolutionary Increase in Neuron Number

First, a few possible, but ultimately unlikely mechanisms should be mentioned. Developmental cell death is extensive, first in the cortical anlage prior to neurogenesis, and later as the neuron layers of post-mitotic cortex settle and make their connections. While these both contribute, the first to the effective size of the founder pool [Rehen et al., 2001], and the second, to the local differentiation of cortical regions [Finlay, 1992], no evidence exists for a systematic relationship between developmental cell death and brain mass on an evolutionary scale. Redirection of the output of founder pools certainly occurs. For example, redirected cells from the medial ganglionic eminence are added to the cortex in monkeys by lateral migration, which increases the complement of inhibitory neurons in the cortex [Letinic et al., 2002]. This is a substantial factor which could distinguish closely related species or taxa, but cannot account for the range of brain volumes over mammals.

The basic parameters of neurogenesis might be altered in three ways to make evolutionarily significant changes in neuron number, considering both single structures and the entire brain. (1) The initial founder pool of stem cells could be increased, or (2) the rate of neurogenesis, neurons produced per unit time, might be increased wholesale or in part, or (3) the duration of cytogenesis/ neurogenesis could be lengthened.

Increase in initial precursor pool number could increase ultimate neuron number, so long as the increased size did not challenge a diffusible molecule range as discussed earlier. For trade-offs in size between "competing" structures, however, this is an attractive hypothesis. We have in fact proposed movement of a developmental boundary as a possible account of the systematic differ-
Finlay 


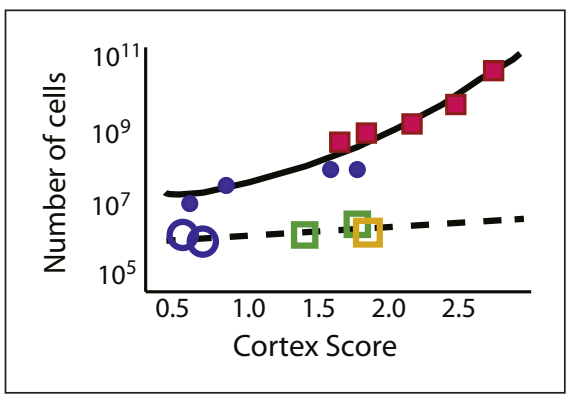

Fig. 1. Estimates for the founder population (dashed line) versus total neuronal output of the ventricular zone (solid line) as a function of "Cortex Score." In the "Translating Time" modeling project [Workman et al., 2013], each species is given a "Species Score" which is a linear ranking of the total duration of its neurodevelopment, compared to other species. The "Species Score" correlates very closely with total brain volume, and was used as a proxy in the case of missing values relevant to the Cahalane et al. [2014] study, to which the reader is referred for the details of this analysis. The Cortex Score similarly combines neurodevelopmental duration to predict volume in this case modified for generation and eventual volume of the cortex alone. The solid symbols represent adult cortical neuron counts in individual rodents (blue disks) and primates (red squares), corrected for developmental neuron death. The open symbols represent empirical counts of cells in the precursor pools of 2 rodents (blue circles), 2 carnivores (green square), and one sheep (orange square). Replotted from Cahalane et al. [2014].

ences in allocation of neuron number to the olfactorylimbic system versus neocortex in major mammalian taxonomic groups. The relevant anlage of the forebrain lateral vesicle might be allocated in changed proportions to one fate or the other. We have found no direct evidence for this hypothesis, however, except that adult neuron numbers in these groups vary reciprocally [Reep et al., 2007].

Considering the cortex alone, as part of an extensive comparative study on rates of fetal brain development, in eutherian mammals, Halley [2017] showed that initial cortical mass as well as subsequent rates of growth are virtually identical to each other across species. Looking more closely at the prior observations of equivalent masses of embryonic zones in animals with extremely different adult cortical volumes, we gathered limited data on cortical plate cell numbers, first, to determine if the relationship of volume and cell number in embryonic zones across species showed any discrepancies, and then, to model the adult ranges of intra- and interspecies variation in cortical neuron number for various ranges of progenitor numbers, cell cycle kinetics, and early cell death [Cahalane et al., 2014]. Specimens from existing collections were used to provide some representative measures to anchor this model, using standard stereological procedures. It was impractical to undertake a systematic statistical study of precursor/adult number relationships, but the cell numbers gathered told the same story as volumes had (Fig. 1). While the slope of precursor numbers plotted against adult brain mass is slightly positive, it is statistically insignificant, with no interesting outliers, and in line with the earlier volumetric studies.

The rate of neurogenesis shows substantial differences, especially between taxonomic groups, while its general envelope and sequence of neuron types stay stable. The cell cycle is rapid at first, and progressively slows. For most eutherian mammals yet studied (including primates, rodents, ungulates, carnivores, and the rabbit), there is no evidence that those with larger brains produce neural tissue at a higher rate [Workman et al., 2013], and their growth curves are superimposable in the range of postnatal days they overlap [Halley, 2017]. For non-eutherian mammals, those marsupials we have investigated, the neurons of the entire brain are produced at a slower rate overall, but with the same sequence and relative intervals as eutherian mammals. Bats and naked mole rats, chosen for investigation because of their atypical longevities for their brain sizes, appear to take a period of time to generate their brains more appropriate to their extended longevities, months rather than the weeks that a mouse would require for its comparable small brain [Finlay and Huang, 2020]. Thus, these two species have a slower rate of neurogenesis, but what feature(s) of their cell cycles slow the rate is not yet known. Occasionally, "blips" of increased cell production on normally smooth neurogenesis curves have been observed [Caviness et al., 2003], but those may reflect second sources of neuron addition to a single adult structure [Letinic et al., 2002].

What has never yet been observed is a wholesale change in the rate of neurogenesis in any structure known to be particularly large in one mammalian species versus another. Such differences in adult brain structure are particularly marked when contrasting the olfactory/limbic system (the olfactory bulb and cortex, hippocampus, other limbic cortex and subcortical structures), versus either "neocortex" or "rest of forebrain" [Finlay and Darlington, 1995; Reep et al., 2007]. When the mechanism of reciprocal specialization on this olfactory/limbic vs. cortex/rest of brain axis has been investigated, the only difference as yet described has been movement of the onset of neurogenesis forward or backward in the relevant structures with respect to each other [Finlay and Darlington, 1995; Workman et al., 2013]. Relative delay of onset of neuro- 
genesis, allowing the founder pool to increase without depletion of the stem cells quitting the founder pool to produce neuroblasts, produces a larger cortex at maturity.

Duration of neurodevelopment is highly correlated with adult brain volume or neuron number with multiple confirming sources [Passingham, 1985; Workman et al., 2013; Halley, 2017]. In our own study of the patterns of neurodevelopment in 18 mammals, including non-eutherian and eutherian mammals, with both highly altricial and precocial species, the absolute length of neurodevelopment, as measured from more than 200 developmental events, was the best predictor of adult brain mass. These mammals progress through a conserved order of developmental milestones, but take allometrically scaled, substantially different absolute amounts of time to execute each step. For example, if a mouse takes 6 days from onset to termination of cortical neurogenesis to produce its small cortex, a more slowly maturing macaque takes minimally about 30 days to generate its larger one.

Constructing Similar Adult Structures with Different Developmental Mechanisms: The Retina in Goldfish and Macaques

We have described here, using primarily evidence from the forebrain, to argue that mammals appear to have a different set of developmental mechanisms to employ in brain construction. Specifically, non-mammals have abundant adult neurogenesis, variable precursor pools, and locally variable rates of neurogenesis, while mammals do not. We can then look at the consequences for the method of construction of an entirely separate structure, to see what the effect of a different developmental "tool chest" might be. The retinas of the goldfish and the monkey both have cones and rods for diurnal and nocturnal vision, a central concentration of cones and related cells for higher acuity vision, and a central-to-peripheral gradient in receptor and neuron density. The goldfish, however, an animal with indeterminate growth, grows its retina throughout its lifespan compared to the one-shot generation of the retina in primates. The goldfish eye may grow so much that a fully functional goldfish hatchling could swim in the globe of an adult goldfish eye [Fernald, 1989].

Goldfish retinal production begins from a vertebrategeneral initial deployment of layers composed of particular neuron types and photoreceptors. After this initial organization step, the goldfish eye continues to generate full new "modules," giving rise to photoreceptors (rods and cones), bipolar cells, retinal ganglion cells, and various interneurons from progenitor pools around its edge, in an annular pattern [Johns, 1979]. Growth is thus partially caused by cell addition at the edges, but the eye also grows by stretching [Hagedorn and Fernald, 1992]. Stretch has the immediate effect of causing the population of rods in the pre-existing retina to fail to tile its surface, compromising the sensitivity of its low-light photon catch. Rods filling these gaps do not migrate from the immature peripheral retina to central retina to fill emerging gaps in the rod surface, but rather are generated from pockets of progenitors committed to rod production studded throughout the retina [Fernald, 1991; Hagedorn et al., 1998]. The goldfish also produces and maintains a horizontally extended area centralis, an area in the temporal retina of increased photoreceptor and neuron density permitting increased acuity, whose angular location in the eye is held roughly constant despite its massive growth and stretch. This perplexing geometric problem is solved by situating a single location with a very high rate of neurogenesis at the edge of the temporal retina [Kwan et al., 1996]. Overall, adult neurogenesis in this teleost can be initiated in response to such diverse factors as overall somatic growth, depletion of particular cell types, and the circadian cycle.

By contrast, diverse mammalian eyes are assembled in single smooth sweep through the fixed order of generation of multipotent retinal progenitors (retinal ganglion cells and cones, then horizontal and amacrine cells, then bipolars and rods). This assembly happens over periods ranging from about 10 to 100 days [Finlay, 2008]. Differences in the numbers of photoreceptor and neuronal classes in different retinal locations for retinas with different adaptive requirements are generated solely by spatially staggered onsets and offsets of cytogenesis in a center-periphery gradient, while the retina is stretching. Features of retinal topography like the cell-packed area centralis or visual streak can also be produced by this mechanism, but the primate fovea does require one or two specific additions to existing mechanisms. The reader is directed to Finlay et al. [2005] for a full description of how only a gradient of onsets and offsets within a conserved order and rate of neuronal and photoreceptor specification are ingeniously orchestrated to produce cone-dense, rod-free foveas, and rod-enriched peripheries in diurnal primates, and a uniformly rod-dense retina in nocturnal ones. These growing primate retinas, unlike the goldfish, harness local differences in maturational state to the elasticity of the retina so that while the eye as a whole stretches in direct response to intraocular pressure (the principal means of eye enlargement in mam- 
mals), the adhesion and connectivity of the earliest-maturing regions are held fixed at their high embryonic densities.

Causes and Consequences of Limitation of

Developmental Mechanisms for Brain Growth

In all vertebrates, including mammals, using diffusible molecules emanating from single sources to polarize and segment germinal zones severely limits variation in their size at this crucial developmental stage. In mammals, the virtually complete restriction of neurogenesis to early development is a restriction on the population of undifferentiated cells that can continue to add neurons to a general pool, or "patch" an emerging problem or perhaps organize a brain feature specific to a maturational state or new niche.

The reasons why mammals might have largely lost adult neurogenesis are unknown, but some limited speculations have their place. Adult neurogenesis can confer some benefits, such as replacement of mechanically or chemically stressed components that lose function, erasure of prior states in mechanisms designed to detect and integrate transitory states, and can permit greater plasticity, as they do in the mammalian olfactory bulb and hippocampus [Ming and Song, 2005]. On the other hand, new neurons added to existing structures in organisms that make extensive use of learning and prediction over a lifetime would seem quite maladaptive, adding noise and systematically destroying the predictions and contingencies laboriously learned.

Interestingly, duration of neurogenesis is a feature represented as a continuous gradient in the fate map of both axes of the embryonic neural plate, rostrocaudal (spinal cord to forebrain) and mediolateral along with neuron type and connectivity specification, and is essential to the regular allometric scaling of vertebrate brains [Finlay et al., 1998; Workman et al., 2013]. The most medial components (somatic and viscero-motoneuron) cease divisions first, then primary sensory neurons, then immediately associated sensorimotor specializations (such as auditory, vestibular, or deep cerebellar nuclei). Integrating structures, such as the cerebellar cortex, midbrain tectum, and the medial, dorsal, and lateral pallium of the forebrain cease division last, and in some cases, never stop. Compared to their forebears, reptiles and birds severely limit adult neurogenesis to those structures derived from the most lateral part of the neural plate, principally the forebrain, and mammals further restrict the process to a subcomponent of the medial pallium and olfactory primordium within the forebrain [Kaslin et al.,
2008]. While the first mammals do not distinguish themselves with obvious features that might make a longer and more stable memory useful, the simple fact of any amount of any period of parental care allow exaptations of this generic ability to permit durable memories of this period, such as who your littermates are or lifetime recognition of nontoxic foods that are only seasonably available.

Looking across the diversity and rapidity of adaptations in the several radiations of fish, which can be catalogued in recent evolutionary history [Kornfield and Smith, 2000] or the demonstrably higher amount of variation in neuron number and density across the avian brain, even with adaptive function altogether undefined [Iwaniuk et al., 2004], the relative simplicity of mammalian brain evolution becomes apparent. With the limited number of developmental mechanisms available for brain construction, mammalian brains may thus become more generic, with fewer ad hoc adaptations, but with an exceptionally large variability in their sizes. The contention in this manuscript is that this often-remarked fact cannot be understood fully by restricting analysis to gene frequencies, or to the models derived from game theory of behavioral ecology. Attention must be paid to the available developmental mechanisms and resulting adult conformations those gene frequencies can actually command.

\section{Large Brains and Long Developmental Duration Force More and More Basic Primate Brain Development to \\ Be Postnatal}

This case is not about conjunctions of mechanistic detail, but rather the pervasive, but imperfect correlation between long durations, large sizes, and primate life history. The developmental allometry arising from these correlations forces much of the basic organization of the primate brain to occur postnatally, in a social context.

Contributing constraints and context:

a. Large bodies, large ranges, long pregnancies, longevity

b. The allometry of neurodevelopment

c. The expansion of the concept of phenotypic integration

\section{Large Bodies, Ranges, Long Pregnancies, and Long} Lives

Large masses, spaces, and durations are positively correlated in the real world, though imperfectly, independent of evolutionary biology, Here, we will consider various correlated aspects of mammalian evolution as an exercise - development, pregnancy and maternal care, life history and long memories and the like. Some of these correlations are reported, some speculative. As discussed 
in the first section, a prolonging duration is how the greater number of neurons composing a large brain is generated ( $r=0.893$ ) [Workman et al., 2013]. Larger bodies correlate with larger brains, more closely than the actual physical requirements of larger sensory surfaces and more muscles would require. Generally, a longer gestation will be required to produce these brains and bodies, and longer postpartum care will be necessary as well (see discussion and regression equations associated with the altricial/precocial distinction in Workman et al. [2013]). If a longer pregnancy and longer postpartum care will be necessary for reproduction, the composition of the immune system should be balanced in favor of increased maternal longevity [Tian et al., 2017]. Larger animals are more likely to have larger home ranges, and migrate longer distances. Larger ranges may permit the discovery of resources like fruits that are patchy in both space and time [Eisenberg and Wilson, 1978; Pagel and Harvey, 1989; DeCasien et al., 2017], but systematically exploiting them may require more long-term memory. If such information is acquired culturally, by the route of imitation, rather than by individual experience alone, the relevant lifespans of young and old must overlap enough for the procedures and routes to be co-experienced, or perhaps taught [Laland, 2004]. Larger brains may assist in the cognitive management of the properties and relationships of larger numbers of individuals [Dunbar et al., 2018]. Determining cause and effect within the patterns of covariation by performing endless regressions on this collection of appositions is not an appealing prospect, but coordination via evolution and development together may eventually provide some simplification.

\section{The Allometry of Neurodevelopment}

One particularly interesting feature of the models of developmental allometry worked out in the "Translating Time" project [Workman et al., 2013] is that for larger and larger brains, more absolute time for "brain construction" is pushed post birth. This is not accomplished by moving birth to an earlier maturational point, which can be seen in altricial rodents like laboratory mice and rats. Moving birth earlier in these small-brained animals born in large litters is unrelated to environmental instruction, as the immaturity of every system limits the usefulness of early birth in acquiring such information. Rather, systematic increase in the relative time allotted to the later events of early brain development such as synaptogenesis, axon pruning, and myelination occurs, involved in activity-related environmental interactions. This relationship is more a mathematical rule than a statistical correlation.
Empirical modeling determines the transformation required to translate the postnatal day of multiple events of neurodevelopment from one species to another beginning from initial neurogenesis and (at the time of this paper) ending around human postnatal day 10 for the full dataset. This can be done with high accuracy, as the fit of data to model is $r=0.9929$ (see www.translatingtime.org). As "Translating Time" is a power function, the relative duration of early and late developmental events varies across species according to their overall developmental length (for example, the relative duration of retinal ganglion cell generation, an early event, will be about 5 days in a mouse and 45 in a human, that is, requiring a 9-fold longer duration, but going from $70 \%$ to $80 \%$ of adult brain mass, a late event that will require about 10 days in a mouse will require not 90 days but nearly 400 in a human, a 40-fold increase.

Doing the math for the range of primate neurodevelopmental durations (corresponding to the range of marmoset to human brain mass) shows a corresponding relationship to the amount of "late" brain development occurring postnatally. First, an average primate birthdate is calculated (acceptable because birth and brain maturational state are independent in the primate samples we have modeled) and referenced to its corresponding developmental stage. Then, the time to a postnatal maturational milepost can be calculated, in this case, the milestone of $80 \%$ of adult brain mass. The absolute and relative number of days from conception to birth, and birth to the designated postnatal milestone, can be calculated over the specified range of primate brain volumes, marmoset to human. This calculation shows that development lengthens in the primates with bigger brains, the percentage of neuromaturational time to reach the $80 \%$ milestone that is postnatal grows from about 40 to $55 \%$.

The kinds of events that are prenatal in primates are linked to fundamental brain construction: all neurogenesis and the formation of brainstem axonal scaffolding; the establishment of primary thalamocortical pathways and the first synapses; and endogenous organizing activity such as retinal waves. Peak synaptogenesis, the majority of synaptic subtraction, and most myelination occurs postnatally. These events are not the final polishing of structural details. The well-known establishment of ocular dominance columns and binocular vision occurs at this point, and additionally, the topographic representation of non-primary cortical areas may be stabilized postnatally [Arcaro and Livingstone, 2017; Imam and Finlay, 2020]. For human infants in their first 6 months of postnatal life, their relative immobility offers an extended pe-
Finlay 
riod with close-up visual inspection of faces, and later, time observing their own manipulation of objects close at hand [Fausey et al., 2016; Smith et al., 2018]. Thus, having a bigger brain automatically entails a greater allowance of time for postnatal learning, no special adaptations necessary. In the anthropological literature, the long childhood of humans is typically assumed to be a special adaptation (for example, Locke and Bogin [2006]), but the evidence presented here would rather suggest a long childhood would be a natural entailment of the slowed postnatal rate of development associated with large brains. This fact does not "explain away" or make human childhood less interesting, but puts human evolution in the matrix of covariation that defines mammalian evolution [Hawkes and Finlay, 2018].

Expansion of the Concept of Phenotypic Integration

Analysis that examines the effect of niche, social structure, environmental affordances, and the like on brain or brain-part mass has generally concerned itself with the partition of variance, for example, showing that increased brain mass in primates is particularly related to frugivory. This means statistically that after shared variance is removed, residual variance in brain size can be accounted for by frugivory [DeCasien et al., 2017]. Problems arise, however, when the statistical account of covariance and residual variance is conflated unnecessarily with the modular account of brain function implied by mosaic accounts of brain evolution. Mosaic brain evolution was originally defined as the possibility for independent evolution of any designated brain part, but is typically used to describe brain regions like thalamic nuclei or cortical areas, and was reserved for cases where a change in size was "allometrically unexpected" [Barton and Harvey, 2000], a distinction that has become progressively lost (for example, Preuss and Wise [2022]). If the residual amount of brain variance after controlling for shared variance, say, $5-15 \%$, is accounted for by frugivory, that does not mean that somehow $5-15 \%$ of the brain is devoted to frugivory [van Schaik et al., 2021]. Perhaps, however, it is not the magnitude of the shared variance that has been miscalculated, but that the concatenation of residual variance with mosaic brain evolution is flawed. Shared or linked mechanisms, from genome, to neurodevelopment to brain circuitry are neglected in this approach.

In other domains, particularly in cranial and skeletal evolution, persistent covariation is now the subject of direct study. In the first studies of this phenomenon, the covariation of elements beak and cranial morphology in

The Contexts of Brain Volume song sparrows and Galapagos finches [Schluter, 1996], the strong claim was made that "Evolutionary response to natural selection cannot ... be predicted from the measurement of phenotypic characteristics alone." In systematic studies of the sources of this structure in variation, termed "adaptive radiation along genetic lines of least resistance," the correlated pattern of variation offered to selection was found not only to account for the shortterm evolutionary likelihood of moving in direction of the correlated variation, but for long-term phenotypic change just as strongly. Studies following on these observations started from these empirical accounts of phenotypic change produced by covariation in beaks and crania. Later, covariation was related to developmental processes, such as duration, rate, or domains of embryonic spatial specification like prosomeres or placodes, or to processes of conflict and adaptation when patterns of covariance might interfere [Besnard et al., 2020; Evans et al., 2021]. With one very interesting exception concerning developmental bias in "the domestication syndrome" [Wilkins, 2020], however, these studies have not reached far into behavior, brain organization, or life history.

The broad classes of covariation listed in the first paragraph of this section were to suggest that for mammals, an important axis of covariation might the small-to-large range of adult brain and body size, to an appropriate short-to-long duration of neurodevelopment and lifespan, concomitant with physiological and immune function adjustments required for each. The consequences of cross-mammalian neurodevelopmental allometry just described, where an increasing proportion of brain development becomes available for postnatal learning in larger and larger brained species is a specific example of such covariation. Faced with changing environments, but which still vary along the general dimensions experienced in their lineage's evolutionary past, adaptation will be eased by such persisting covariation, "evolvability."

Genetic linkage of such predictive covariation is not without cost, if low-probability environments should be encountered. For example, an energetically costly overbuilt brain in a simple environment will spend resources best used elsewhere. Earlier, two exceptions to the general relationship of brain mass to longevity were mentioned, the bat and naked mole rat, and they might demonstrate such a cost. We found that while these species decoupled brain size and longevity (that is, both have long lives and small brains), the length of their neurodevelopment corresponded to their excess longevity, not their small brains [Finlay and Huang, 2020]. These two species generate their small brains in exceptionally long times, 
taking the 2-3 months it would be necessary to generate a cat brain, for example, to generate their mouse-sized brains, which typically requires less than a month. These two species show that it is possible to decouple developmental features that usually covary, which is not surprising, as covariation does not entail permanent linkage. In this case, only the linkage of duration of neurodevelopment and brain mass seen in most other mammals became decoupled, while the "life history" features of extended early development remain coupled. The generally accepted account of the extended longevity of birds, and by extension, the general category of flying species, is that their greater longevity compared to non-flying species is due to lessened lifelong mortality due to decreased predation. The lesser mortality is not equal throughout the lifespan, however: for birds, predation is high in the nest and then falls to very low levels, well below most land mammals, when nestlings can fly. Therefore, shortening the maximally perilous pre-flight period of development would be appear very desirable. Even so, the bats retain an extended duration of helpless early development to build mouse-sized brains. A possible explanation of the bat's coupling of extended lifetime longevity with unnecessarily extended neurodevelopmental duration (in terms of the absolute time necessary to generate a brain of a bat's size), is that these two features are genetically coupled because of their usual covariation in mammalian life history. The variation permitting greater longevity comes coupled to neurodevelopmental length, adaptive variation along the genetic lines of least resistance.

\section{Conclusion}

In this paper, we have discussed two extended contexts for understanding the phenotypic characteristic of a species possessing a certain brain mass. In all of this discussion, the assumption has been that possession of a brain, large or small, is adaptive. The goal in this case is to give specific examples of how changing repertoires of developmental mechanisms both constrain and direct eventual brain structure, both through the close-up lens of embryological mechanisms and the broader view of life history.

Summarizing briefly, the first context considered the developmental mechanisms that both directly control, or impinge upon the total number of neurons a species might be able to generate for its brain. An example of a developmental constraint was laid out: that using diffusible molecules to specify the early polarity of a two-di- mensional embryonic array (the cortical plate) constrained its size and made it unlikely that substantial increase in the initial number of cells in that primordial zone could be a mechanism of increasing adult cortex neuron number. We then considered how a variable feature of vertebrates, adult neurogenesis, might interact with that constraint. Adult neurogenesis varies across vertebrate phylogeny: fish (bony and cartilaginous) and amphibians are capable of substantial neurogenesis throughout their brains, throughout their lifespan; most reptiles and bird taxa restrict adult neurogenesis to large forebrain regions; mammals restrict adult neurogenesis to two small, discrete forebrain regions. The conjunction of the two features in mammals, the use of diffusible molecules in embryonic specification, and very restricted neurogenesis in adulthood results in that changing duration of neurogenesis dominates all other mechanisms for alteration of mammalian brain mass. Turning the example around, the consequences of the mammalian mechanistic restriction to duration can be seen in how the similar features of the adult teleost and mammalian retina are produced by quite different mechanisms. At the end, the most interesting challenge to consider is how the dominating feature of developmental duration in mammalian brain evolution may have canalized mammalian evolution. An adaptive challenge for behavior might be met quite differently in teleosts than mammals. For example, while extra neurons for a sensory demand can be produced on demand in particular locations at particular life stages, as in acquisition of dominant male status in the cichlid Haplochromus burtoni [Fernald, 2012], in humans, no newly-promoted CEO has been demonstrated to be thereby in possession of more neurons in any location. In mammals, such contingencies must be met with existing neurons with preformation or lifetime plasticity. Do the same essential features arise through different mechanisms, as did in their retinas, or are the features changed by the mechanisms available?

The second context looked at brain size through a wider developmental focus. Again, principally considering mammals, larger brains have a strong statistical relationship with multiple features of life history. Development, from infancy to adolescence will be longer, parental care must be correspondingly lengthened; increased body size, increased spatial ranges are all high, though imperfect correlates of large brains. In morphological development, the interesting genetic causes and genetic consequences of such covariation is a rapidly growing field of study. Understanding of the genetics of the structure and pace of life history has hardly begun, but some of the un- 
usual regularities of neurogenesis in the mammalian brain might provide a way forward.

In the beginning of this article, we noted predictable features of how scientists approach new complex subjects, first mapping concrete, statable aspects of one domain of inquiry onto another, discussing genes for aggression, particular brain areas for language, then considering their linear interactions. As knowledge is gathered, every domain has become more multivariate, statistical, multi-stable, and better described by network architectures. Mathematics and models for these complex mappings have grown very quickly, and perhaps occasionally, too quickly. This article attempted to take one phenotypic feature, brain size, which has many contestants vying to account for its adaptive features. Instead, we have examined the brain from the view of the control and integration of the developmental mechanisms that makes the neurons that compose it, and from the broad view of life history in which any large or small brain is embedded. The project of the extended evolutionary synthesis is embodied in this integration.

\section{Conflict of Interest Statement}

The authors have no conflicts of interest to declare.

\section{Funding Sources}

There was no funding for this study, except as cited in the earlier papers from this laboratory.

\section{Author Contributions}

Barbara Finlay is the sole author.

\section{References}

Altman J, Das GD. Autoradiographic and histological studies of postnatal neurogenesis I. A longitudinal investigation of the kinetics, migration and transformation of cells incorporating tritiated thymidine in neonate rats, with special reference to postnatal neurogenesis in some regions. J Comp Neurol. 1966 (126):337-90.

Alvarez-Buylla A. Neurogenesis and plasticity in the CNS of adult birds. Exp Neurol. 1992;115: $110-4$.

Anderson ML. After phrenology: neural reuse and the interactive brain. MIT Press; 2014.

Anton-Bolanos N, Lopez-Bendito A, Espinosa López-Bendito G. Developmental interactions between thalamus and cortex: a true love reciprocal story. Curr Opin Neurobiol. 2018; 52:33-41. http://dx.doi.org/ 10.1016/j.conb. 2018.04.018.

Arcaro MJ, Livingstone MS. A hierarchical, retinotopic proto-organization of the primate visual system at birth. eLife. 2017;6:e26196.

Assimacopoulos S, Kao T, Issa NP. Grove EA Fibroblast growth factor 8 organizes the neocortical area map and regulates sensory map topography. J Neurosci. 2012;32(21):7191201. https://doi.org/10.1523/JNEUROSCI. 0071-12.2012.

Balasubramanian R, Zhang X. Mechanisms of FGF gradient formation during embryogenesis. Semin Cell Dev Biol. 2016;3:94-100. https: //doi.org/10.1016/j.semcdb.2015.10. 004.

Barton RA, Harvey PH. Mosaic evolution of brain structure in mammals. Nature. 2000;405: $1055-8$.
Bernard A, Lubbers LS, Tanis KQ, Luo R, Podtelezhnikov AA, Finney EM, et al. Transcriptional architecture of the primate neocortex. Neuron. 2012;73(6):1083-99. http://dx.doi. org/ 10.1016/j.neuron.2012.03.002.

Besnard F, Picao-Osorio J, Dubois C, Félix MA. A broad mutational target explains a fast rate of phenotypic evolution. eLife. 2020;9:e54928. http://dx.doi.org/ 10.7554/eLife.54928.

Burke AC, Nelson CE, Morgan BA, Tabin C. Hox genes and the evolution of vertebrate axial morphology. Development. 1995; 121(2): 333-46. http://dx.doi.org/ 10.1242/dev.121.2. 333.

Cadwell CR, Bhaduri A, Mostajo-Radji MA, Keefe MG, Nowakowski TJ. Development and arealization of the cerebral cortex. Neuron. 2019; 103(6): 980-1004. https://doi. org/10.1016/j.neuron.2019.07.009.

Cahalane DJ, Charvet CJ, Finlay BL. Modeling local and cross-species neuron number variations in the cerebral cortex as arising from a common mechanism. Proc Natl Acad Sci USA. 2014;111(49):17642-7. http://dx.doi. org/ 10.1073/pnas.1409271111.

Caviness VS, Goto T, Tarui T, Takahashi T, Bhide PG, Nowakowski RS. Cell output, cell cycle duration and neuronal specification: a model of integrated mechanisms of the neocortical proliferative process. Cerebral Cortex. 2003; 13(6):592-8. https://doi.org/10.1093/cercor/ 13.6.592.

Cohen JR, d'Esposito M. The segregation and integration of distinct brain networks and their relationship to cognition. J Neurosci. 2018; 36(48):12083-12094. http://dx.doi.org/ 10.1523/ jneurosci.2965-15.2016.
Damasio AR, Geschwind N. The neural basis of language. Ann Rev Neurosci. 1984;7:127-47.

Darbellay F, Bochaton C, Lopez-Delisle L, Mascrez B, Tschopp P, Delpretti S, et al. The constrained architecture of mammalian Hox gene clusters. Proc Natl Acad Sci USA. 2019; 116(27):13424-33. http://dx.doi.org/ 10.1073/ pnas. 1904602116.

de Visser JA, Krug J. Empirical fitness landscapes and the predictability of evolution. Nat Rev Genet. 2014;15(7):480-90.

DeCasien AR, Williams SA, Higham JP. Primate brain size is predicted by diet but not sociality. Nat Ecol Evol. 2017;1(5):1-7. http://dx.doi. org/ 10.1038/s41559-017-0112.

Dunbar RIM, Mac Carron P, Shultz S. Primate social group sizes exhibit a regular scaling pattern with natural attractors. Biol Lett. 2018; 14(1):20170490.

Eisenberg JF, Wilson DE. Relative brain size and feeding strategies in the Chiroptera. Evolution. 1978;32:740-51.

Evans KM, Larouche O, Watson SJ, Farina S, Habegger ML, Friedman M. Integration drives rapid phenotypic evolution in flatfishes. Proc Natl Acad Sci. 2021;118(18): e2101330118. http://dx.doi.org/ 10.1073/pnas. 2101330118.

Fausey CM, Jayaraman S, Smith LB. From faces to hands: changing visual input in the first two years. Cognition. 2016;152:101-7. https:// doi.org/10.1016/j.cognition.2016.03.005.

Fernald RD. Seeing through a growing eye. In: Carew TJ, Kelly DB, editors. Perspectives in neural systems and behavior. MBL lectures in biology. Liss; 1989. p. 151-74. 
Fernald RD. Retinal rod neurogenesis. In: Finlay BL, Sengelaub DR, editors. Development of the vertebrate retina. Plenum Press; 1991. p. $31-42$.

Fernald RD. Social control of the brain. Annu Rev Neurosci. 2012;35(1):133-51.

Finlay BL, Darlington RB. Linked regularities in the development and evolution of mammalian brains. Science. 1995;268(5217):1578-84.

Finlay BL. Cell death and the creation of regional differences in neuronal numbers. J Neurobiol. 1992;23(9):1159-71.

Finlay BL. The developing and evolving retina: using time to organize form. Brain Res. 2008; 1192:5-16.

Finlay BL, Hersman MN, Darlington RB. Patterns of vertebrate neurogenesis and the paths of vertebrate evolution. Brain Behav Evol. 1998; 52(4-5):232-42.

Finlay BL, Huang K. Developmental duration as an organizer of the evolving mammalian brain: scaling, adaptations, and exceptions. Evol Develop. 2020;22(1-2):181-95. http:// dx.doi.org/ 10.1111/ede.12329.

Finlay BL, Silveira LCL, Reichenbach A. Comparative aspects of visual system development. In: Kremers J, editor. The structure, function and evolution of the primate visual system. John Wiley and Sons; 2005. p. 37-72.

Frankenhuis WE, Walasek N. Modeling the evolution of sensitive periods. Dev Cogn Neurosci. 2020;41:100715.https://doi.org/10.1016/j. dcn.2019.100715

Furusawa C, Kaneko K. A dynamical-systems view of stem cell biology. Science. 2012; 338(6104):215-7.

Hagedorn M, Fernald RD. Retinal growth and cell addition during embryogenesis in the teleost, Haplochromis burtoni. J Comp Neurol. 1992; 321:193-208

Hagedorn M, Mack AF, Evans B. Fernald RD The embryogenesis of rod photoreceptors in the teleost fish retina, Haplochromis burtoni. Dev Brain Res. 1998;108(1-2):217-27. https: //doi.org/10.1016/S0165-3806(98)00051-0.

Hawkes K, Finlay L. Mammalian brain development and our grandmothering life history. Physiol Behav. 2018;193:55-68.

Halley AC. Minimal variation in eutherian brain growth rates during fetal neurogenesis. Proc Biol Sci. 2017;284(1854):20170219.

Holland PW, Marlétaz F, Maeso I, Dunwell TL, Paps J. New genes from old: asymmetric divergence of gene duplicates and the evolution of development. Phil Trans Roy Soc B. 2017; 372(1713): 20150480. http: //dx.doi.org/ 10.1098/rstb.2015.0480.

Hu Y, Linz DM, Parker ES, Schwab DB, Casasa S, Macagno ALM, et al. Developmental bias in horned dung beetles and its contributions to innovation, adaptation, and resilience. Evol Devel. 2020;22(1-2):165-80. http://dx.doi. org/ 10.1111/ede.12310.

Hubel DH, Wiesel TN. TN Receptive fields and functional architecture of monkey striate cortex. J Physiol. 1968;195:215-43. https://doi. org/10.1113/jphysiol.1968.sp008455.
Imam N, Finlay BL. Self-organization of cortical areas in the development and evolution of neocortex. Proc Natl Acad Sci. 2020;11:724. http://dx.doi.org/ 10.1073/pnas.2011724117.

Isler K, Van Schaik CP. How humans evolved large brains: comparative evidence. Evol Anthropol. 2014;23(2):65-75.

Iwaniuk AN, Dean KM, Nelson JE. A mosaic pattern characterizes the evolution of the avian brain. Proc Roy Soc Lond B. 2004;271:S14851. https://doi.org/10.1098/rsbl.2003.0127.

Jablonka E, Lamb M. Evolution in four dimensions. Cambridge, MA, USA: MIT Press; 2005.

Johns PR. Growth and neurogenesis in adult goldfish retina. In: Freeman D, editor. Developmental neurobiology of vision. Series AR. Nato ASI Series Plenum; 1979. p. 345-57.

Kanwisher N, McDermott J, Chun MM. The fusiform face area: a module in human extrastriate cortex specialized for face perception. J Neurosci. 1997;17(11):4302-11. https://doi. org/10.1523/JNEUROSCI.17-11-04302.

Kaslin J, Ganz J, Brand M. Proliferation neurogenesis and regeneration in the non-mammalian vertebrate brain. Philos Trans Roy Soc B. 2008; 363: 101-22. https://doi.org/10.1098/ rstb.2006.2015.

Kirschner MW, Gerhart JC. The plausibility of life: resolving Darwin's Dilema. Yale University Press; 2005.

Kornfield I, Smith PF. African cichlid fishes: model systems for evolutionary biology. Ann Rev Ecol Syst. 2000;31(1):163-96. https://doi. org/10.1146/annurev.ecolsys.31.1.163.

Kwan JW, Lee MJ, Mack AF, Chiu JF, Fernald RD. Nonuniform distribution of cell proliferation in the adult teleost retina. Brain Research. 1996; 712(1):40-4. https://doi.org/10.1016/ 0006-8993(95)01426-8.

Lai YT, Yeung CKL, Omland KE, Pang EL, Hao $\mathrm{Y}$, Liao BY, et al. Standing genetic variation as the predominant source for adaptation of a songbird. Proc Natl Acad Sci. 2019;116(6): 2152-7. https: //doi.org/10.1073/pnas. 1813597116.

Laland KN. Social learning strategies. Learn Behav. 2004;32(1):4-14.

Laland KN, Uller T, Feldman MW, Sterelny K, Müller GB, Moczek A, et al. The extended evolutionary synthesis: its structure, assumptions and predictions. Proc Roy Soc B. 2015; 282(1813): 1019.

Laughlin DC, Messier J. Fitness of multidimensional phenotypes in dynamic adaptive landscapes. Trends Ecol Evol. 2015;30(8):487-96.

Lein ES, Belgard TG, Hawrylycz M, Molnár Z. Transcriptomic perspectives on neocortical structure, development, evolution, and disease. Ann Rev Neurosci. 2015;40:629-52. http://dx.doi.org/ 10.1146/annurev-neuro070815-013858.

Letinic K, Zoncu R, Rakic P. Origin of GABAergic neurons in the human neocortex. Nature. 2002;417(6889):645-9.
Lewicki MS, Olshausen BA, Surlykke A, Moss CF. Scene analysis in the natural environment. Front Psychol. 2014;5:199. http://dx.doi.org/ 10.3389/fpsyg.2014.00199.

Locke JL, Bogin B. Language and life history: a new perspective on the development and evolution of human language. Behav Brain Sci. 2006;29:259-325. https://doi.org/10.1017/ S0140525X0600906X.

MacIver MA, Finlay BL. The neuroecology of the water-to-land transition, and the evolution of the vertebrate brain. Philos Trans R Soc Lond B Biol Sci. 2021 Dec 27;377(1844):20200523. https://doi.org/10.1098/rstb.2020.0523.

MacIver MA, Schmitz L, Mugan U, Murphey TD, Mobley $\mathrm{CD}$. Massive increase in visual range preceded the origin of terrestrial vertebrates. Proc Natl Acd Sci. 2017;114(12):E2375-84. http://dx.doi.org/ 10.1073/pnas.1615563114.

Ming GL, Song H. Adult neurogenesis in the mammalian central nervous system. Annu Rev Neurosci. 2005;28(1):223-50.

Molnár Z, Kaas JH, de Carlos JA, Hevner RF, Lein E, Němec P. Evolution and development of the mammalian cerebral cortex. Brain Behav Evol. 2014;83(2):126-39. http://dx.doi.org/ $10.1159 / 000357753$.

Newman SA. Form and function remixed: developmental physiology in the evolution of vertebrate body plans. J Physiol. 2014;592(11): 2403-12.

Noble D, Jablonka E, Joyner MJ, Müller GB, Omholt SW. Evolution evolves: physiology returns to centre stage. J Physiol. 2014;592(11): 2237-44. http://dx.doi.org/ 10.1113/jphysiol.2014.273151

Olshausen BA, Field DJ. Emergence of simple-cell receptive field properties by learning a sparse code for natural images. Nature. 1996; 381(6583):607-9. https://doi.org/10.1038/ $381607 \mathrm{a} 0$.

Pagel MD, Harvey PH. Taxonomic differences in the scaling of brain weight on body weight in mammals. Science. 1989;244:1589-93.

Parker HJ, Krumlauf R. Segmental arithmetic: summing up the Hox gene regulatory network for hindbrain development in chordates. Develop Biol. 2017;6(6):e286. https:// doi.org/10.1002/wdev.286.

Parsons KJ, Albertson RC. Unifying and generalizing the two strands of evo-devo. Trends Ecol Evol. 2013;28:584-991. http://dx.doi.org/10.1016/ j.tree.2013.06.009.

Passingham RE. Rates of brain development in mammals including man. Brain Behav Evol. 1985;26:167-75.

Preuss TM, Wise SP. Evolution of prefrontal cortex. Neuropsychopharmacol. 2022;47(1):319. http://dx.doi.org/ 10.1038/s41386-02101076-5.

Puelles L. Thoughts on the development, structure and evolution of the mammalian and avian telencephalic pallium. Philos Trans R Soc Lond B Biol Sci. 2001;356:1583-98.

Puelles L. Pallio-pallial tangential migrations and growth signaling: new scenario for cortical evolution? Brain Behav Evol. 2011;78(1):108-27. 
Puelles L, Harrison M, Paxinos G, Watson C. A developmental ontology for the mammalian brain based on the prosomeric model. Trends Neurosci. 2013;36(10):570-8. http://dx.doi. org/10.1016/j.tins.2013.06.004.

Ramaekers A, Claeys A, Kapun M, MouchelVielh E, Potier D, Weinberger S, et al. Altering the temporal regulation of one transcription factor drives evolutionary trade-offs between head sensory organs. Devel Cell Bio. 2019; 50(6): 780-92.e7. https://doi.org/10.1016/j. devcel.2019.07.027.

Ramírez JC, Marshall JAR. Can natural selection encode Bayesian priors? J Theor Biol. 2017; 426: 57-66. https://doi.org/10.1016/j.jtbi. 2017.05.017.

Reep R, Darlington RB, Finlay BL. The limbic system in mammalian brain evolution. Brain Behav Evol. 2007;70:57-70. https://doi.org/ $10.1159 / 000101491$.

Rehen SK, McConnell MJ, Kaushal D, Kingsbury MA, Yang AH, Chun J. Chromosomal variation in neurons of the developing and adult mammalian nervous system. Proc Natl Acd Sci. 2001;98(23):13361-6. https://doi.org/ $10.1073 /$ pnas. 231487398

Rubenstein JLR, Shimamura K, Martinez S, Puelles L. Regionalization of the prosencephalic neural plate. Annu Rev Neurosci. 1998;21: 445-77. https://doi.org/10.1146/annurev.neuro. 21.1.445.

Schluter D. Adaptive radiation along genetic lines of least resistance. Evolution. 1996;50(5): 1766-74.
Slodkowicz G, Goldman N. Integrated structural and evolutionary analysis reveals common mechanisms underlying adaptive evolution in mammals. Proc Natl Acad Sci. 2020;117(11): 5977-86. http: //dx.doi.org/ 10.1073/ pnas.1916786117.

Smith LB, Jayaraman S, Clerkin E, Yu C. The developing infant creates a curriculum for statistical learning. Trends Cogn Sci. 2018;22(4): 325-36. https: //doi.org/10.1016/j. tics.2018.02.004.

Smulders TV, Gould KL, Leaver LA. Using ecology to guide the study of cognitive and neural mechanisms of different aspects of spatial memory in food-hoarding animals. Philos Trans R Soc Lond B Biol Sci. 2010;365(1542): 883-900.

Song S, Liu L, Edwards SV, Wu S. Resolving conflict in eutherian mammal phylogeny using phylogenomics and the multispecies coalescent model. Proc Natl Acd Sci. 2012;109(37): 14942-7. https: //doi.org/10.1073/pnas. 1211733109 .

Sporns O. Networks of the brain. Cambridge, MA, USA: MIT Press; 2011.

Striedter GF, Northcutt RG. Brains through time: a natural history of vertebrates. Oxford University Press; 2020.

Tian X, Seluanov A, Gorbunova V. Molecular mechanisms determining lifespan in shortand long-lived species. Trends Endocrinol Metab. 2017;28(10):722-34.

van Schaik CP, Triki Z, Bshary R, Heldstab SA. A farewell to EQ: a new brain size measure for comparative primate cognition. Brain Behav Evol. 2021;96:1-12. https://doi.org/10.1159/ 000517013 .
Vermeij GJ. How the land became the locus of major evolutionary innovations. Curr Biol. 2017;27(20):3178-82.e1.

Watanabe K, Stringer S, Frei O, Umićević Mirkov M, de Leeuw C, Polderman TJC, et al. A global overview of pleiotropy and genetic architecture in complex traits. Nature Genetics. 2019; 51(9): 1339-1348. https://doi.org/ 10.1038/s41588-019-0481-0.

West-Eberhard MJ. Developmental plasticity and evolution. Oxford University Press; 2003.

Wilkins AS. A striking example of developmental bias in an evolutionary process: the "domestication syndrome”. Evol Dev. 2020;22(1-2): 143-53.

Wilson DS, Sober E. Reintroducing group selection to the human behavioral sciences. Behav Brain Sci. 1994;17(04):585-608. http://dx.doi. org/ 10.1017/S0140525X00036104.

Wilson DS, Van Vugt M, O'Gorman R. Multilevel selection theory and major evolutionary transitions: implications for psychological science. Curr Direct Psych Sci. 2008;17(1):69. http: //dx.doi.org/10.1111/j.1467-8721. 2008.00538.x

Workman AD, Charvet CJ, Clancy B, Darlington RB, Finlay BL. Modeling transformations of neurodevelopmental sequences across mammalian species. J Neurosci. 2013;33(17): 7368-83. http://dx.doi.org/ 10.1523/jneurosci.5746-12.2013.

Ypsilanti AR, Rubenstein JLR. Transcriptional and epigenetic mechanisms of early cortical development: an examination of how Pax6 coordinates cortical development. J Comp Neurol. 2016;524(3):609-29. http://dx.doi. org/10.1002/cne.23866. 